\title{
CRYOGENIC AIR COOLING TECHNIQUE FOR MACHINING OF SUPER ALLOYS
}

\begin{tabular}{|c|c|c|c|}
\hline M.E. Mohana Sundaram, & M. Radha Krishna, & S. Maria Arockiam, & M. Sanmuganantham, \\
Senior Technical officer & Principal Technical officer & Senior Technician-3 & Technician-2 \\
Propulsion division & Propulsion division & Propulsion division, & Propulsion division, \\
CSIR-National Aerospace & CSIR-National Aerospace & CSIR-National Aerospace & CSIR -National Aerospace \\
Laboratories, Bangalore, & Laboratories, Bangalore, & Laboratories, Bangalore, & Laboratories, Bangalore, \\
India & India & India & India \\
mohana_s@ @ nal.res.in & krishmr@ nal.res.in & arockiam@nal.res.in & msananth23@nal.res.in \\
& & & \\
\end{tabular}

\begin{abstract}
-
This paper discusses about the development of effective air cooling technique for machining the hard materials. In this technique the cold air at $-140^{\circ} \mathrm{C}$ is used, this is achieved by passing the air through a tube which is immersed in liquid nitrogen. The cold air is used during metal cutting in place of a flooding coolant. Here 3 types of Nozzles are designed to pass the chilled air onto the cutting zone. These nozzles are calibrated by using the Twin Beam Shadowgraph Technique to obtain optimized distance of flow of air without any divergence. Experiments were carried out to measure the Air and Tool temperatures during machining. Results show smooth machining, less machining time, more feed rate, increase in tool life. At the same time this technique will reduce the production cost when compared with other existing cooling techniques. Thus it can be an alternative tool cooling technique in place of harmful/hazardous liquid-based cooling.
\end{abstract}

Keywords-air cooling; gas cooling; cryogenic cooling; alternative cooling; Green environment cooling; etc.

\section{INTRODUCTION}

\section{Green Environment:}

Cooling and lubrication have important roles in reducing the severity of the contact conditions at the cutting tool-work piece interfaces. The use of coolant / lubricant fluids or 'Wet cutting' is particularly common in Aerospace and Automotive industries. Even after usage, the disposal of contaminated cutting fluids necessitates some treatment and hence the cost of disposal and danger of environmental pollution.

In recent years there are many researches in the area of innovative cutting lubricants for manufacturing industries to control the cost of manufacturing, maintain a pollution free and healthy working zone [1].

U.S. Dixit et al [2], through the research states that the performance of gaseous coolants are better than the liquid coolants in many cases. Moreover, there is no waste disposal problem as the gases can be released to atmosphere.

Cryogenic cooling approaches are introduced in the field of machining to overcome some of the issues by Boswell et al, [3]. Stanford M et al [4] have investigated the use of gaseous and liquid nitrogen as a cutting fluid, results indicate that a $55 \%$ reduction in flank wear is achievable when turning BS 970-080A15 (En32b).

Hong Shane Y., et al [5] in their approach have compared the Cryogenic machining with conventional dry cutting and cutting with emulsion cooling. The study found that cooling approaches in the order of effectiveness (worst to best).

Akshaya T Poojary et al, [6] in their investigation have dipped the work piece in cryogenic liquid (LN2) for a specified period of time and then fixed on to the chuck for turning. In their conclusion for Cryogenic cooling it was observed that as the cutting speed increased better surface finish of the work piece is obtained. Cryogenic cooling showed decrease of surface roughness to a significant extent for high feed rates compared to dry and wet machining due to the property of LN2 to reduce the cutting zone temperature and thereby causing easy flow of chips through the tip of the tool.

The Cryogenic Machining has many advantages [7] such as saving Time, Tools and hence Money, Surface integrity and Part quality (Reduction of the White Layer / Alpha Layer, Reduction of Residual stress, Reduction in Burr formation, Reduction of Surface distortion (Grain Boundary Distortion)), Increased processing speed, Decreased Tool wear, Environmental-friendly Green manufacturing, No more Hazardous coolants, Reduced overheads and Sustainable manufacturing.

\section{AIR COOLING}

One of the interesting researches in Cryogenic machining technology is Air cooling. According to Brian Boswell [8], [3], the introduction of Ranque-Hilsch Vortex tube to provide cold air to the tool interface has shown to significantly improve the performance of machining, a highly significant reduction in tool tip temperature, thereby reduced flank wear and increased tool life is achieved. It is possible to obtain temperatures as low as $-46^{\circ} \mathrm{C}$ and as high as $+127^{\circ} \mathrm{C}$ when using the Ranque-Hilsch vortex tube.

Use of refrigerated gases was examined by Walter [9], he showed that the use of these fluids can be economic in certain 


\section{Asia Pacific Journals}

situations. Hollis [10], found that carbon dioxide can be particularly effective in reducing Crater wear on Carbide tools when machining Titanium alloys and other difficult to machine materials.

Comparison of using refrigerated compressed air with ambient compressed air was conducted by Pahlitzsch [11], where he found that there is a 400 percent increase in tool life if the air is at $-40^{\circ} \mathrm{C}$ to $-56^{\circ} \mathrm{C}$, while even at $-8^{\circ} \mathrm{C}$ a 40 percent increase occurred when the tests were carried out on steel, machined at $30 \mathrm{~m} / \mathrm{min}$.

Since Pahlitzsch proved that refrigerated air increases tool life, the challenge is to provide a very cold stream of air to the tool tip during machining. The rate of Heat dissipation at the tool tip when cooled by air is limited in comparison to liquid coolant.

It is very clear that the air cooling technique have many advantages when compared to the liquid coolant.

In present attempt, we have enhanced the cooling of the air up to a range of $-120^{\circ} \mathrm{C}$ to $-150^{\circ} \mathrm{C}$ by passing the air through a coiled Copper tube dipped in Liquid Nitrogen, kept in an insulated box. The normal compressed air while passing through a Copper coil (dipped in $\mathrm{LN}_{2}$ bath), loses its temperature and then exits the insulated tube and nozzle to reach the tool. We have developed nozzles, as shown in fig.1, in order to focus the air to the required cutting edges of the tool, to increase the gap between the nozzle and tool as required while machining of the materiel. Nozzle III is selected as it meets the above requirements (FP3 of Fig.1).

\section{DEVELOPMENT OF NOZZLE \& EXPERIMENT}

Nozzle is a device used for tool cooling with relatively larger nozzle-tool gap / distance. While machining with air cooling the position of the Nozzle plays a very important role. Researcher Shane Y. Hong [12] quote that LN2 cooling with optimized nozzle position improved the tool life by $57 \%$ compared to flood cooling. In order to focus the air to the required cutting surface, we have developed three nozzles with unique features and hence different flow patterns were obtained.

The flow patterns obtained from Twin-beam shadowgraph flow visualization technique are as shown in Fig.1. The nozzle I gives a flow with large divergence when compared to nozzle II and III. The nozzle II with the elliptical feature parallel to the tool axis, gives flow diversion angle of $24^{\circ}$ to the air, about a length of $9.5 \mathrm{~mm}$ with same cross sectional area as shown in Fig.1 (FP2a). When the elliptical feature of nozzle II is horizontal i.e. perpendicular to the tool axis (shown in Fig.1 (FP2)), gives flow without diversion in vertical plane, about the length of 10.5 to $26.70 \mathrm{~mm}$ with same cross sectional area, shown in Fig.1 FP2a \& FP2. The Nozzle III, with circular edge, gives flow without diversion for a length of $71.0 \mathrm{~mm}$ to $100 \mathrm{~mm}$ with same cross sectional area is as shown in Fig.1 (FP3). Hence nozzle III is selected for the present study.

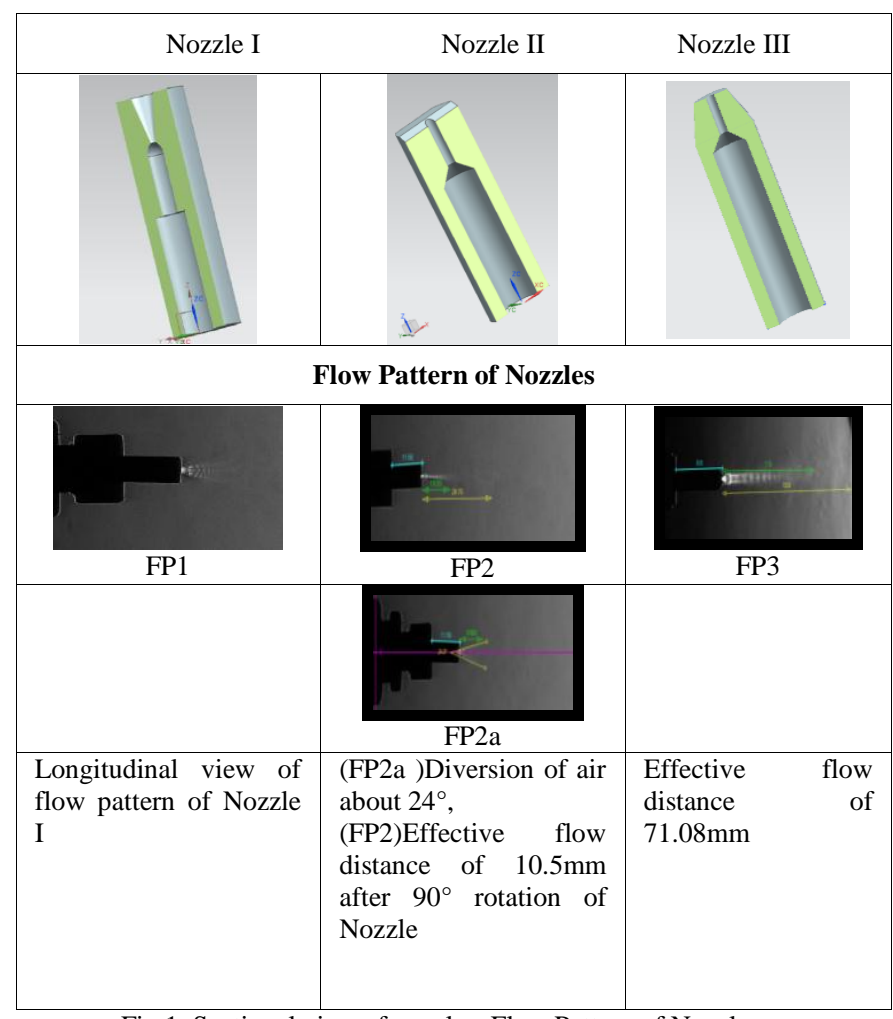

Fig.1. Sectional view of nozzles, Flow Pattern of Nozzles.

\section{Experimental Condition:}

It is necessary to find the relation between the Tool temperature, Distance (Nozzle - Tool gap) and Air Pressure so as to have minimum possible Tool temperature while machining.

The schematic of Experimental set up is shown in Fig. 2. Nozzle is fixed horizontally facing the tool, the inlet air pressure can be controlled and varied by using pressure control valve. Also the distance between the Nozzle - Tool gap can be varied by moving the nozzle, measured by a scale. The experiments are carried out on Carbide tools which has thermal conductivity of $110 \mathrm{~W} /\left(\mathrm{m}^{\circ} \mathrm{K}\right)$. The Tool temperature for various Distances (Nozzle - Tool gap) and Pressures are measured.

\section{EXPERIMENTAL SETUP (AIR COOLING) FOR MATERIAL MACHINING}

Coiled Copper tube (Refrigeration coil) dipped in Liquid Nitrogen:

The schematic of experimental setup is shown in Fig.2. This setup has the following elements in the cooling system. 1) Compressor 2) Control valve and throttles 3) Insulated Copper tube 4) Refrigeration coil and Liquid nitrogen(LN2) in an Insulated container 5) Pressure gauge 6) Nozzle 


\section{Asia Pacific Journals}

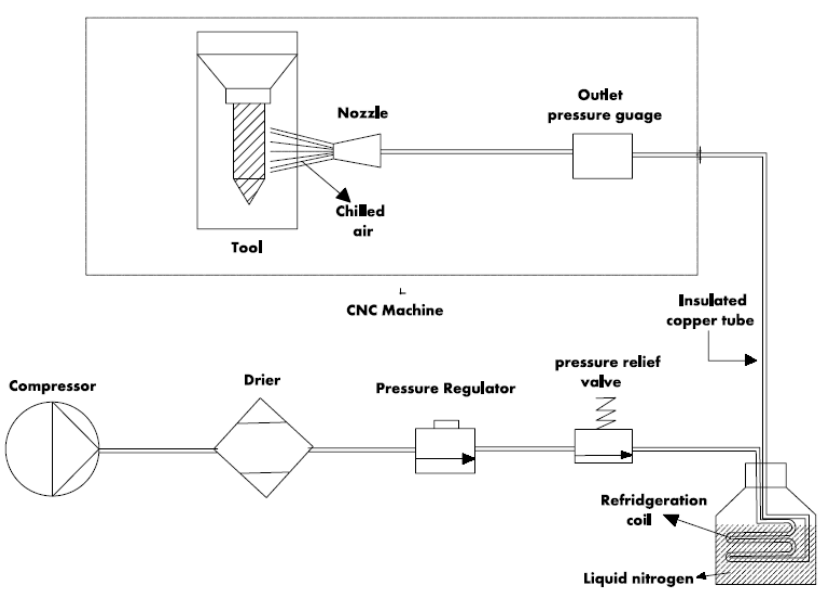

Fig.2. Schematic of Experimental setup.

The moisture content in compressed air should be removed by passing through a drier to eliminate the precipitation of moisture as ice in the Copper tube and Pressure gauge will cause variation in flow rates and pressure which in turn reduces the effective cooling of the tool. It is also noticed that there is a drastic increase in temperature because of the blocking of the outlet by the ice crystals leading to rise in pressure, hence melting of ice around the nozzle and inside the copper tubing. The cooling of tool also depends on the tool conductivity.

We obtained this chillness in air by passing compressed air through a number of coils dipped in the liquid nitrogen and the whole above system is kept in a non conducting box. As by law of heat transfer, the heat from the air is transferred to liquid nitrogen thereby cooling the air and the difference of the temperature between these two is almost $220^{\circ} \mathrm{C}$.

\section{EXPERIMENT}

The experiment has been carried out on Carbide tools which has a thermal conductivity of $110 \mathrm{~W} /\left(\mathrm{m}^{\circ} \mathrm{K}\right)$. The tool should be selected according to the work piece because different tools give different reaction while cutting and some specific tools only can provide us the intended operation over the work piece. For instance, we selected Carbide type tool. as shown in Fig.4

\begin{tabular}{|c|c|c|c|}
\hline \multicolumn{4}{|c|}{$\begin{array}{l}\text { Diameter }=2 \mathrm{~mm} \text {, Tool material }=\text { Carbide } \\
\text { Nozzle-tool gap }=3.5 \mathrm{~mm} \text {, Exposure time }=10 \mathrm{sec} \text { to } 30 \mathrm{sec}\end{array}$} \\
\hline S1.No. & Pressure(Psi) & Air Temp $\left({ }^{\circ} \mathrm{C}\right)$ & Tool Temp $\left({ }^{\circ} \mathrm{C}\right)$ \\
\hline 1. & 4 & -137 & -0.9 \\
\hline 2. & 8 & -141 & -23 \\
\hline 3. & 12 & -153 & -49 \\
\hline
\end{tabular}

TABLE.I TOOL TEMPERATURE VS. NOZZLE-TOOL GAP AND PRESSURE.

\begin{tabular}{|c|c|c|}
\hline \multicolumn{3}{|c|}{$\begin{array}{l}\text { Diameter }=2 \mathrm{~mm} \text {, material }=\text { Carbide, Pressure }=8 \text { Psi } \\
\text { Tool Exposure time }=10 \mathrm{sec} \text { to } 30 \mathrm{sec}\end{array}$} \\
\hline S1 No. & Nozzle-Tool Gap(mm) & Tool Temp $\left({ }^{O} \mathrm{c}\right)$ \\
\hline 1. & 35 & 10 \\
\hline 2. & 15 & 6 \\
\hline 3. & 8 & -0.3 \\
\hline
\end{tabular}

TABLE.II NOZZLE-TOOL GAP(MM) VS TOOL TEMPERATURE

Milling operation:

Machining operation on Nimonic 90 has been carried out using DMU 50 Deckel Maho CNC Milling Machine, as shown in Fig.3

\section{Cutting Tool Description:}

Designation of tool

SGS make Tool diameter $=8 \mathrm{~mm}$,

Shank diameter $=8 \mathrm{~mm}$,

No. of flutes $=4$,

Helix angle $=30^{\circ}$,

Flute length $=20 \mathrm{~mm}$,

Overall length $=60 \mathrm{~mm}$

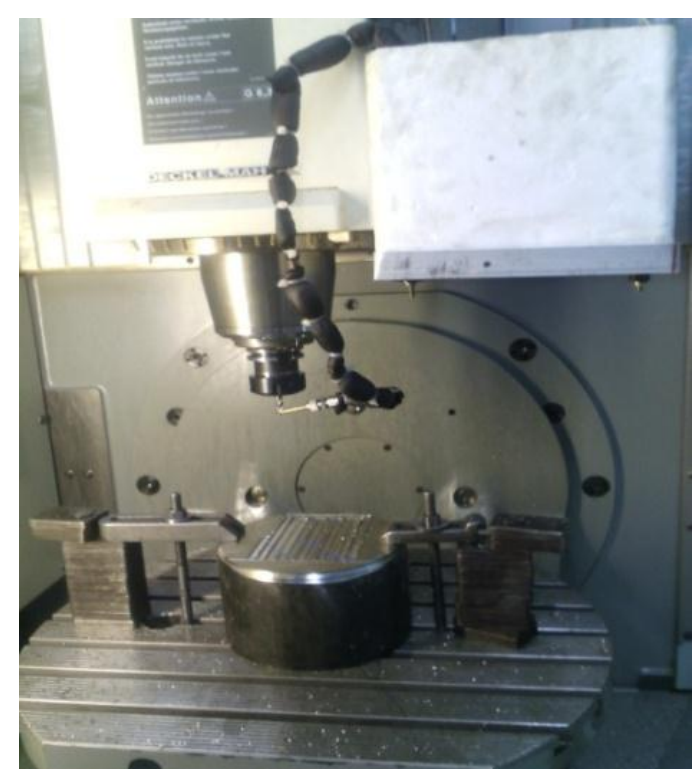

Fig.3. Nimonic 90 work piece.

Experimental Conditions for Milling:

Different cooling techniques under same feed, depth of cut, cutting speed and tool material. 


\section{Asia Pacific Journals}

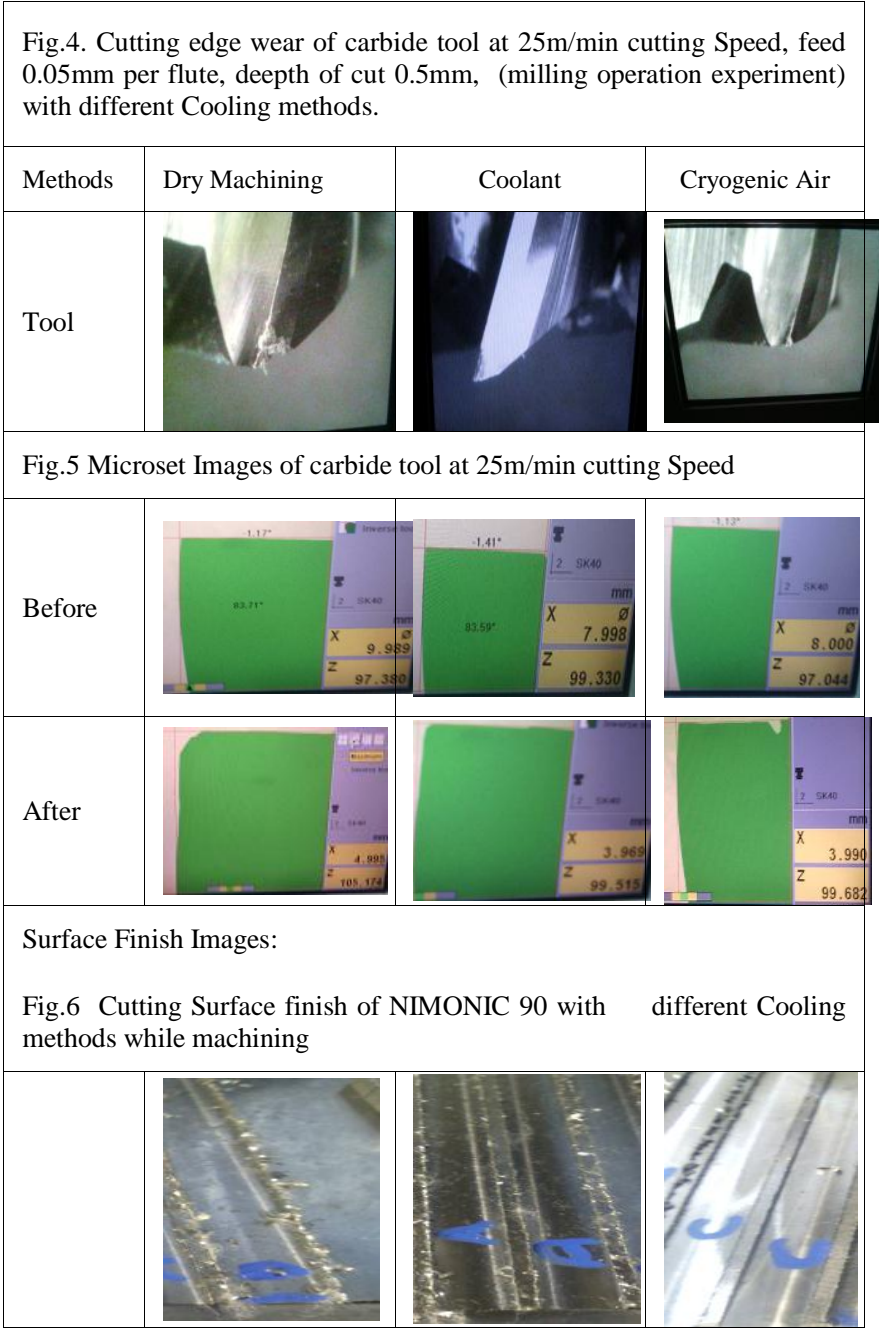

After machining under dry, liquid coolant and cold air methods, the specimens have been sent to metrology and metallurgical laboratories. It is in process of examining the SEM images of microstructure, micro hardness, tool wear and surface roughness.

Here an approximate wear of each tool bit is obtained by analyzing tool before and after machining with the help UNO115 MICROSET, as shown in fig.5. Cutting edge wear is more for Dry machining and with coolant machining when compared with Cryogenic Air cooling method.

The Surface finish images of NIMONIC 90 workmaterial is as shown in Fig.6. Surface finish of Chilled air cooling technique gives cutting surfaces without any burrs and with good quality.

\section{RESULTS AND DISCUSSION}

1. Output from our attempt using liquid nitrogen is really good. The air temperature range we obtained from this setup is of $-120^{\circ} \mathrm{C}$ to $-150^{\circ} \mathrm{C}$, which has the potential to provide excellent tool life and better surface finish.
In view of industry, production cost:

2. Comparing in perspective of production cost, the cryogenic liquid nitrogen cooling method [12] utilizes 4.8 Liters per hour while cryogenic chilled air requires only 2.5 Liters per hour taking tool life from both methods is considerably same.

3. CNC, Conventional machine tools can easily adapt the Cryogenic Air Cooling technique because it is an external circuit and will never affect the life of the machine spindle and other parts, with the same initial investment a better production, profit and reduction in production time can be obtained.

\section{CONCLUSIONS}

1. Cold air cooling technique provides good tool life compared to liquid coolant and dry machining.

2. Cold air cooling is economical compared to the direct cryogenic liquid nitrogen spraying technique since 2 liters of LN2/hour is only required for cold air while 4.8 liters of LN2/hour is required for cryogenic liquid nitrogen spraying.

3. Cold air cooling technique gives cutting surfaces without any burrs and with good quality.

4. More the flow rate of the chilled air over the tool, more the cooling of tool.

5. Tool cooling is inversely proportional to the nozzletool gap distance.

\section{Acknowledgement}

The authors are thankful to Director, CSIR - National Aerospace Laboratories, Bangalore and Mr. P. Manjunath, Head, Propulsion Division for giving this Opportunity. The authors are also thankful to Y. Giridhar babu, Mr. R. Prathapanayaka and team, Propulsion Division for their guidance and encouragements for under taking this study and in writing this paper. Authors also acknowledge the colleagues of Special purpose Workshop, Propulsion Division, Raghu kumar H.S. for giving timely help during the process of machining and other activities.

\section{References}

[1] wikipedia

[2] U.S. Dixit et al., Environmentally Friendly Machining, Springer Briefs in Applied Sciences and Technology, DOI 10.1007/978-1-4614-23089_4, 2012.

[3] Boswell, Brian and Chandratilleke, Tilak T. 2009. "Air-Cooling Used For Metal Cutting". American Journal of Applied Science. 6 (2): pp. 251-262

[4] Stanford M. ; Lister P. M. ; Morgan C. ; Kibble K. A. ; (2009) "Investigation into the use of gaseous and liquid nitrogen as a cutting fluid when turning BS 970-80A15 (En32b) plain carbon steel using WCCo uncoated tooling". Journal of materials processing technology 


\section{Asia Pacific Journals}

ISSN 0924-0136 Source / Source 2009, vol. 209, no2, pp. 961972 [12 page(s) (article)]

[5] Hong Shane Y. ${ }^{(1)}$; Yucheng Ding ${ }^{(1)}$; "Cooling approaches and cutting temperatures in cryogenic machining of Ti-6Al-4V" International journal of machine tools \& manufacture ISSN 0890-6955 CODEN IMTME3, 2001, vol. 41, nº10, pp. 1417-1437 (26 ref.)

[6] Akshaya T poojary, Rajesh nayak "Investigation of surface roughness in machining of AISI 1040 steel" International journal of mechanical engineering and technology (ijmet), volume 5, issue 9, September (2014), pp. 36-43

[7] http://www.onlinetmd.com/tmd1114-cryogenic-machiningicefly.aspx\#.VNQ_C6GHK

[8] Brian, Boswell "use of air cooling and its effectiveness in dry machining processes", thesis is presented for the degree of doctor of philosophy of Curtin university of technology, June 2008
[9] L. Walter, "Carbon dioxide as cutting tool coolant repays research with imposing savings," Canadian machining and metalworking, vol. 76, p. 94, 1965. References 175

[10] W. S. Hollis, "The application and effect of controlled atmospheres in the machining of metals", Int. J. Mach. Tool des. and Res., vol.1, p.59, 1961.

[11] G. Pahlitzsch, "Gases are good cutting coolants," American machinist, vol. 97, p. 196, 1953.

[12] Shane Y. Hong, New cooling approach and tool life improvement in cryogenic machining of Ti-6Al-4V 